\title{
Oscillating ultra-cold neutron spectrometer
}

\author{
D. Rozpedzik ${ }^{1, \text { a }}$, K. Bodek ${ }^{1}$, B. Lauss ${ }^{2}$, D. Ries ${ }^{3}$, P. Schmidt-Wellenburg ${ }^{2}$, and G. Zsigmond ${ }^{2}$ \\ ${ }^{1}$ Marian Smoluchowski Institute of Physics, Jagiellonian University, Cracow, Poland \\ ${ }^{2}$ Paul Scherrer Institute, Villigen, Switzerland \\ ${ }^{3}$ Institute of Nuclear Chemistry, Johannes Gutenberg University, Mainz, Germany
}

\begin{abstract}
The energy spectrum of ultra-cold neutrons (UCN) is very often a key point to determine the systematic effects in precision measurements utilizing UCN. The proposed novel method allows the insitu measurements of the UCN velocity distribution and its time evolution. In addition, the proposed UCN spectrometer can be a handy diagnostic tool for monitoring the UCN spectrum in critical places in the transport system connecting an UCN source with experiments. In this paper, we present the preliminary results from measurements and simulations using the oscillating UCN spectrometer at the PSI UCN source.
\end{abstract}

\section{Introduction}

The field of fundamental physics utilizing neutrons has experienced a significant increase in activity over the last two decades. Extraordinary sensitivity and precision of results obtained in experiments using slow neutrons to fundamental particle physics, astrophysics, and cosmology, have prompted investments in the intense cold- and ultra-cold neutron sources in a number of leading centers in the world [1-10]. In many cases, these experiments provide information complementary to existing accelerator-based nuclear physics facilities or high-energy accelerators [11-16].

UCNs are neutrons which are so slow that they are reflected under any angle of incidence from suitable materials and hence can be stored. Typical materials therefore restrict the UCN velocities to about $7 \mathrm{~m} / \mathrm{s}$ [11]. One of the most important properties of UCN used in experiments is the possibility of storing them in traps, where their velocity distribution is slowly evolving due to interacions with imperfect surfaces. The insitu measurement of the UCN velocity spectrum and its evolution in time is important in many precision experiments such as e.g. a measurement of the neutron electric dipole moment (nEDM). A direct measurement of the energy distribution of stored $\mathrm{UCN}$ is a key ingredient for proper estimation of a number of systematic effects occurring in such experiments. Until now, a convenient method of the direct measurement of the energy has not yet been known. The measurement of energy can be performed by measuring the UCN velocity distribution while knowing (or assuming) the phase space distribution.

The commonly used techniques are e.g. time-of-flight spectrometry using choppers, transmission through foils with different Fermi potentials as cut-offs, or measuring UCN in the gravitational field at different heights for highly collimated beams [17-19]. The most frequently used approach to cope with this problem of velocity

$\bar{a}$ e-mail: dagmara.rozpedzik@uj.edu.pl determination utilizes the UCN acceleration in the Earth gravitational field such that even the slowest UCN can pass the Fermi potential barrier of the detector entrance window. This is achieved by changing the UCN detector height relatively to the height of the UCN storage volume [20,21]. Such a method is cumbersome and introduces new systematic effects arising from scattering off variable length UCN guides and energy dependent transmission. Wider application of this method e.g. in the study of the UCN spectrum evolution is hardly possible. In this situation, the shape of the energy spectrum must be deduced from Monte Carlo simulation and is uncertain since several and critical parameters influencing the UCN energy distribution in the storage volume change in time in an uncontrolled way. Recent results from the spinecho method [22] developed at PSI demonstrated an insitu energy spectrum measurement for specific situations when the spin procession of an UCN ensemble can be manipulated with RF signals. However, this method is hardly applicable for routine diagnostics and may be used only in special cases. Moreover, it needs an independent verification.

So far an efficient and routine way of measuring the UCN velocity distribution is unknown. The method proposed in this project will allow for a direct measurement of the UCN velocity components normal to the detector surface covering the range $(0-7) \mathrm{m} / \mathrm{s}$ in a single scan. The spectrometer can be oriented in any direction so that all three components can be measured individually and hence the full velocity distribution in the probed volume can be converted into the kinetic energy distribution. The new idea assumes using a moving detector which provides the time-dependent effective Fermi potential (sensed by UCN in their rest frame) instead of e.g. accelerating UCN in the gravitational field prior to detection. The advantage of the proposed method is that it can be applied to any direction whereas gravitation affects only the vertical UCN velocity component. If the entire range of the UCN velocity component will be covered in one run, then the whole spectrum will be measured at 
identical conditions and it will be possible to study its evolution. Such a method provides a diagnostic tool for experiments where knowledge of the neutron spectrum is important.

The paper is organized as follows. In Sect. 2 the proposed oscillating detector method for the measurement of the UCN velocity spectrum is described. Construction of the oscillating UCN spectrometer prototype is presented in Sect. 3. The results from Monte Carlo simulations are discussed in Sect. 4. Section 5 includes a preliminary analysis of the first sample of collected data at the West-1 port of the UCN source at the Paul Scherrer Institute, Villigen, Switzerland [23]. Finally, Sect. 6 contains conclusions and outlook.

\section{Method}

In the first approximation, a slow neutron approaching a material surface experiences a repulsive or attractive force due to Fermi pseudo-potential. This force is perpendicular to that surface. In consequence, the interaction changes only the momentum component perpendicular to the surface at the point of incidence. An incident neutron will penetrate through the barrier only, if its velocity component perpendicular to the surface is greater than the Fermi velocity $v^{\mathrm{F}}$ of the material surface. In this approximation, we neglect tunneling phenomena.

The basic idea of the oscillating UCN spectrometer is to use the mechanical acceleration of the detector towards incoming neutrons. At the moment of hitting the detector's active surface moving with the velocity $-v_{\mathrm{d}}$, the potential barrier is exceeded for neutrons with the velocity component parallel to the detector motion $v_{n}>v_{\mathrm{d}}^{\mathrm{F}}-v_{\mathrm{d}}$. The moving detector changes the relative perpendicular velocity of the neutron which may be interpreted as a change of the Fermi potential. The spectrometer geometry is sketched in Fig. 1 (left panel). The linear oscillations are realized with a mechanical crankshaft. If the detector oscillates in a way that the appropriate range of relative velocities is covered, all UCNs velocities get a chance to be registered. Simultaneously, the acquisition system stores the information at what detector velocity $v_{\mathrm{d}}$ this registration took place, in other words, what was the effective Fermi velocity at the instant of registration. In this way, the effective Fermi velocity will be recorded for all UCN detected in a single scan so that the minimum detection velocity can be assigned to all of them. The knowledge of the detector velocity at instant of the UCN registration is crucial for the decomposition method (see Sect. 5) from which the velocity spectrum will be obtained.

The detector attached to a mechanical crankshaft driven by a constantly rotating axis oscillates harmonically. The detector position is given by the equation

$$
z_{\mathrm{d}}=A+L-(A \cos \alpha+L \cos \beta),
$$

where $A$ is the radius of the crankshaft i.e. the oscillation amplitude, $L$ is length of the connecting rod and the angles $\alpha$ and $\beta$ are defined in Fig. 1 (left panel). Using the law of sines we get the relation

$$
A \sin \alpha=L \sin \beta
$$

then

$$
\sin \beta=\lambda \sin \alpha,
$$

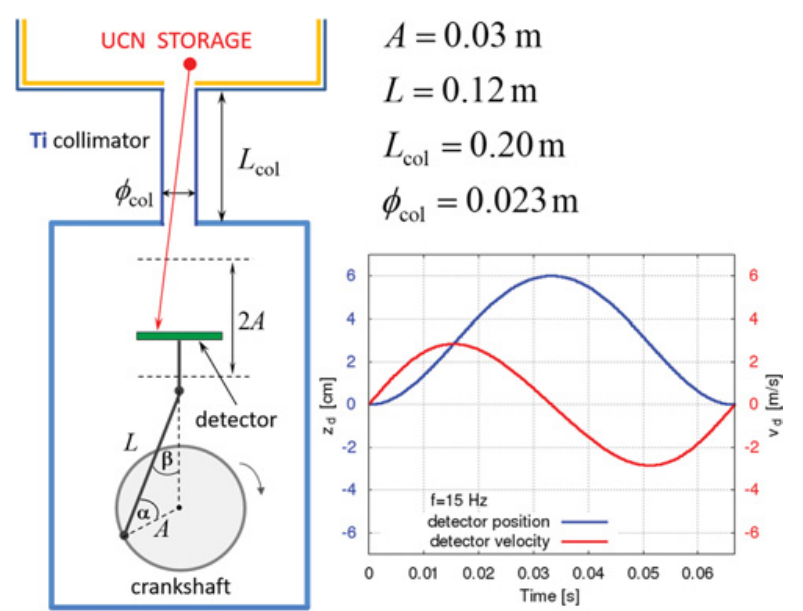

Figure 1. General conception of the UCN oscillating spectrometer. Left panel: Crankshaft oscillator. The blue line marks the UCN absorbing materials: the titanium collimator with negative Fermi potential $\left(V_{\mathrm{col}}^{\mathrm{F}}=-45 \mathrm{neV}\right)$ and the aluminium walls of chamber with Fermi velocity $v_{\mathrm{Al}}^{\mathrm{F}}=3.2 \mathrm{~m} / \mathrm{s}$. The yellow line identifies the UCN reflector with Fermi velocity $\left(v_{\mathrm{s}}^{\mathrm{F}}=\right.$ $6.3 \mathrm{~m} / \mathrm{s}$ ) typical for storage vessel. The green box denotes the detector surface with Fermi velocity $v_{\mathrm{d}}^{\mathrm{F}}=4.0 \mathrm{~m} / \mathrm{s}$. Right panel: Time dependence (within one oscillation period) of the detector position $z_{\mathrm{d}}$ and detector velocity $v_{\mathrm{d}}$.

where $\lambda=A / L<<1$ is the ratio of the radius of the crankshaft to the length of the connecting rod. Applying mathematical transformations

$$
\begin{aligned}
\cos \beta & =\sqrt{\left(1-\sin ^{2} \beta\right)}=\sqrt{\left(1-\lambda^{2} \sin ^{2} \alpha\right)} \\
& \approx 1-0.5 \lambda^{2} \sin ^{2} \alpha=1-\frac{\lambda^{2}}{4}(1-\cos 2 \alpha) .
\end{aligned}
$$

By inserting Eq. (4) into Eq. (1) and using the relation $\alpha=2 \pi f t$, Eq. (1) can be written (in time dependence) as

$$
z_{\mathrm{d}}(t)=A\left((1-\cos (2 \pi f t))+\frac{\lambda}{4}(1-\cos (4 \pi f t))\right)
$$

where $f$ is the frequency of the movement. The detector velocity is a first derivative of the detector position (Eq. (5)) so that

$$
v_{\mathrm{d}}(t)=2 \pi f A\left(\sin (2 \pi f t)+\frac{\lambda}{2} \sin (4 \pi f t)\right) .
$$

The time dependence of the detector position and detector velocity is illustrated in Fig. 1 (right panel). It is easy to see from Eq. (6) that in order to cover the velocity range of the UCN $(0-7) \mathrm{m} / \mathrm{s}$ in a half period and if $v_{\mathrm{d}}^{\mathrm{F}}$ is zero, the product $A \cdot f$ should be about $1 \mathrm{~m} / \mathrm{s}$. At a fixed oscillation amplitude $A$, one can define the required $\mathrm{UCN}$ velocity range by changing the oscillation frequency $f$ and respecting the offset introduced by the Fermi velocity $v^{\mathrm{F}}$ of the detector materia $v_{\mathrm{d}}^{\mathrm{F}}$. The first measurement was performed with the Fermi velocity of the detector surface $v_{\mathrm{d}}^{\mathrm{F}}=4 \mathrm{~m} / \mathrm{s}$ (see Sect. 3) as indicated in Fig. 1. For available maximum frequency $f=15 \mathrm{~Hz}$ and the amplitude $A=0.03 \mathrm{~m}$, the detector velocity covered the range $v_{\mathrm{d}} \in(-2.83,2.83) \mathrm{m} / \mathrm{s}$. This allows for registrations of UCN with a minimum velocity of $1.17 \mathrm{~m} / \mathrm{s}$. 


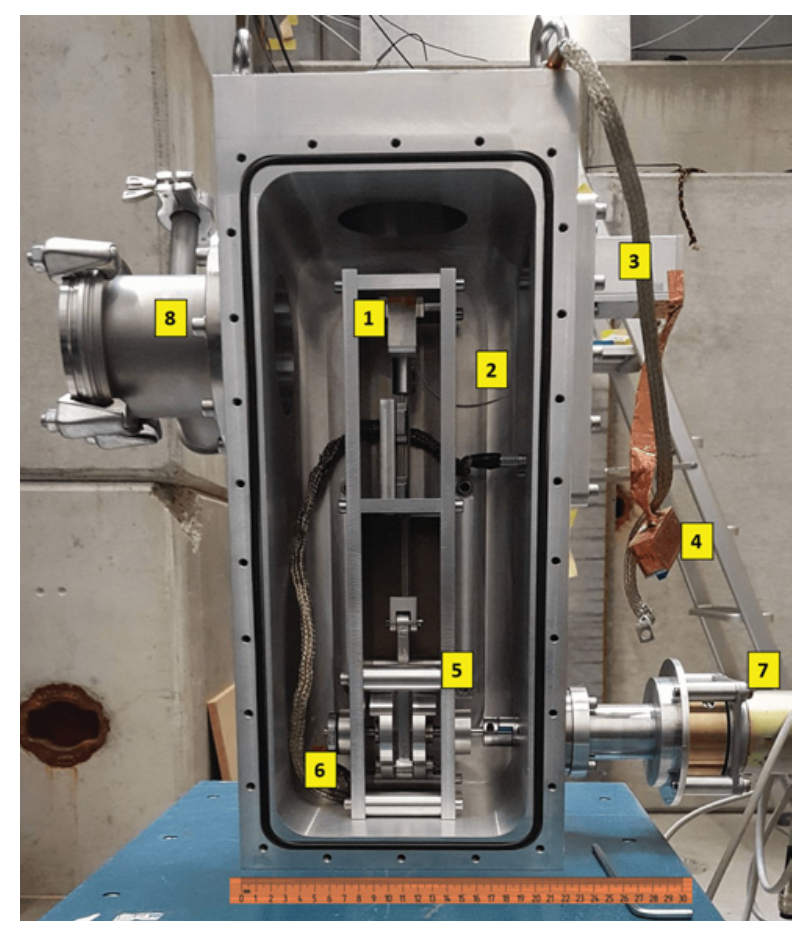

Figure 2. Oscillating UCN Spectrometer (OTUS): 1) detector head; 2) moving connection cable; 3) preamplifier box; 4) connecting cable to the interface module; 5) crankshaft mechanism; 6) driving motor synchronization sensor; 7) DC motor; 8) vacuum flange.

\section{Spectrometer construction}

UCNs are typically transported in vacuum of about a few times $10^{-6}$ mbar. Therefore the oscillating detector must also be located in vacuum. For this purpose, a dedicated chamber was constructed with a vacuum tight rotating feedthrough. The detector was placed inside the chamber together with the crankshaft mechanism that drives the detector oscillations with the fixed amplitude. The chamber was connected to the neutron guide through a collimator made of a titanium tube which allowed to get an almost parallel UCN beam hitting directly the oscillating detector. Tests have shown that a vacuum level in the $10^{-6}$ mbar range was achieved when this chamber with the oscillating detector was pumped by a turbomolecular pump. Figure 2 shows the spectrometer without the front cap of the vacuum chamber. The most important parts are described in the caption.

The detector head consists of a Li-glass scintillator read out by an MPPC array of $6 \times 6 \mathrm{~mm}^{2}$ sensors [24], (see Fig. 3) connected with the front-end electronics located outside the vacuum chamber. Such a combination permits the construction of a small and lightweight assembly able to move at variable speeds covering the UCN velocity range. The MPPC consist of $14 \mathrm{SiPM}$ diodes operated at $2 \mathrm{~V}$ over the minimum breakdown voltage adjusted individually. At these conditions, a single photoelectrons count is translated to about $2 \mathrm{mV}$ signal amplitude at an electronic noise level (FWHM) of less than $0.5 \mathrm{mV}$. A scope screen shot presenting the amplitude distribution of dark counts is shown in Fig. 3. The detector can sustain an oscillating frequency of at least $f=15 \mathrm{~Hz}$ without losing the signal resolution achievable when it is operated at rest.

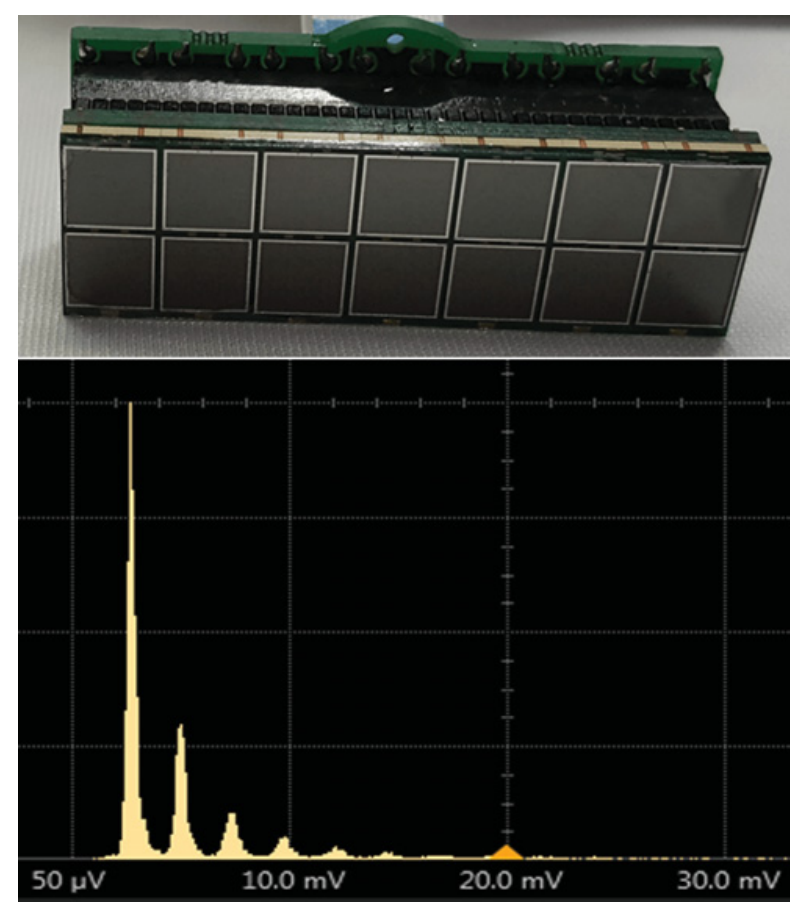

Figure 3. MPPC array. Upper panel: MPPC composed of the single SiPM sensors (its total size is about $15 \times 45 \mathrm{~mm}$ ). Bottom panel: Scope screen-shot of the dark count spectrum of the oscillating Hamamatsu SiPM read out by custom made preamplifier.

The UCN detection is based on the ${ }^{6} \mathrm{Li}$ neutron capture reaction:

$$
n+{ }^{6} \mathrm{Li} \rightarrow{ }^{3} \mathrm{H}(2.74 \mathrm{MeV})+{ }^{4} \mathrm{He}(2.05 \mathrm{MeV}) .
$$

The $100 \mu \mathrm{m}$ thick natural ${ }^{6} \mathrm{Li}$ content glass scintillator (GS10 [25]) was successfully used for UCN e.g. in Ref. [26]. The small thickness reduces the sensitivity to thermal neutron capture and to $\gamma$-ray induced background. Additionally, $\gamma$-ray counts are rejected by the Pulse-Shape Discrimination (PSD) method [27] since $\gamma$-ray signals do not show a slow decaying component and are therefore shorter than the scintillation signals in the Li-glass by recoiling $\alpha$-particles and tritons. The PSD parameter is calculated according to:

$$
P S D=\frac{\int_{t_{0}}^{t_{\mathrm{L}}} Q_{\mathrm{L}}(t) d t-\int_{t_{0}}^{t_{\mathrm{S}}} Q_{\mathrm{S}}(t) d t}{\int_{t_{0}}^{t_{\mathrm{L}}} Q_{\mathrm{L}}(t) d t}
$$

where $Q_{\mathrm{S}}$ denotes the charge collected in the short time gate $\left(t_{\mathrm{S}}\right)$ and $Q_{\mathrm{L}}$ corresponds to the total charge collected in the long time gate $\left(t_{\mathrm{L}}\right)$. The initial time $t_{0}$ is the start of charge integration. It is calculated for each event separately based on the rise time detection which is identical for both neutron and gamma signals. Examples of signals registered by the MPPC are shown in Fig. 4. The indicated times correspond to those of Eq. (8).

The crankshaft is driven by a DC motor (M) synchronized with the data acquisition system (DAQ). The basic scheme of the DAQ is presented in Fig. 5. A dedicated interface module (IM) was constructed to couple the front end part serving the SiPM diodes with a digitizer. The IM consists of the precision power supply unit, the trigger logic (TRG) and the DC motor 


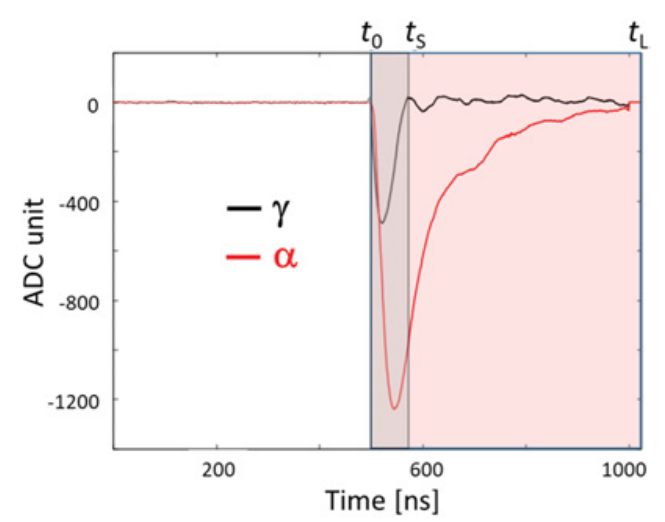

Figure 4. Examples of signals delivered by the MPPC. The red line corresponds to a typical signal produced by an $\alpha$ particle while the black one refers to a typical signal from $\gamma$ background. The times $t_{0}, t_{\mathrm{S}}, t_{\mathrm{L}}$ are defined in Eq. (8).

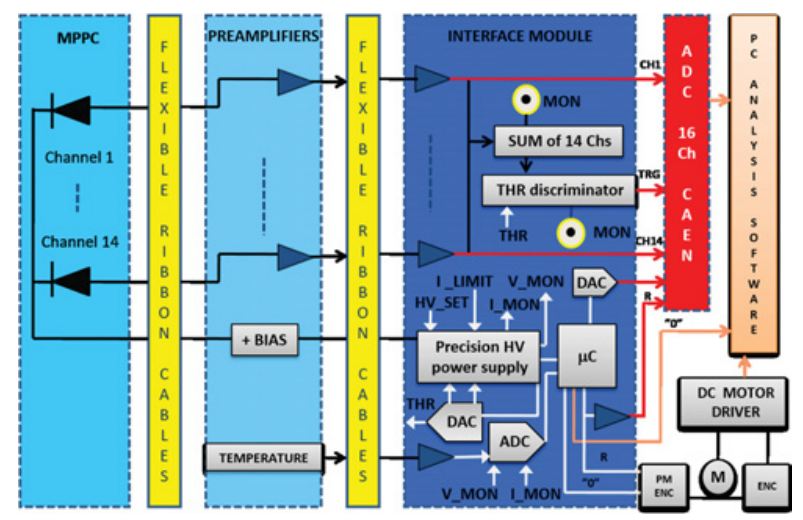

Figure 5. Structure and data flow of the data acquisition system. Details are described in the text.

driver unit. In the trigger part, all analog signals from the preamplifiers are added and fed to the threshold discriminator which produces a TTL trigger pulse for the analog-to-digital converter (ADC) [28]. Independently, the analog signals from the SiPM preamplifiers are transmitted to the digitizer's inputs. The digitized data are transmitted to a PC over a USB link. The MPPC is powered by a precision voltage supply unit controlled by a slow-controller interface which allows for setting the discriminator threshold (THR) and configuration parameters for the DC motor driver unit. The DAQ provides time stamping of the detector signals and for monitoring the motor rotation phase (phase encoder PM ENC). Time stamps of the detector signals allow to uniquely deduce at what speed UCN was detected and relate this information to the minimum $\mathrm{UCN}$ velocity necessary for crossing the moving-detector, effective Fermi-potential barrier.

\section{Monte Carlo simulation}

Monte Carlo simulations are necessary for understanding and optimization of the oscillating UCN spectrometer. In particular, it is important to estimate the size of effects potentially deteriorating the original $\mathrm{UCN}$ velocity distribution as the UCN reflected from a moving surface change their kinetic energy. Some of them may return back to the storage and try to enter the spectrometer again. We identify these effects as kinetic heating and/or kinetic
Table 1. Simulated frequencies and corresponding velocity range.

\begin{tabular}{cc}
\hline $\mathrm{f}[\mathrm{Hz}]$ & detector velocity range $[\mathrm{m} / \mathrm{s}]$ \\
\hline 15 & {$[1.2-6.8]$} \\
10 & {$[2.1-5.9]$} \\
5 & {$[3.0-4.9]$} \\
\hline
\end{tabular}

cooling. It turned out that they are completely negligible, if the dimensions and materials of the spectrometer are properly chosen. Practically, the most important condition is to assure that UCNs reflected from the detector surface are absorbed by surrounding surfaces and have no chance to collide with the detector again.

Another important role of the simulations is to provide the spectrometer response functions (called here the detector velocity profiles). The detector velocity profile describes the transmission of a given UCN velocity bin (at the collimator entrance) and its distribution as a function of the detector velocity $v_{\mathrm{d}}$ at fixed oscillation frequency $f$. The velocity profiles are needed for the decomposition of the measured total distribution (as a function of the detector velocity) into the velocity bins of the original UCN spectrum as described in the next chapter.

Simulations respect the spectrometer geometry, including collimator, fixed oscillation frequency and properties of materials such as Fermi velocity, fraction of Lambert diffuse reflections and loss probability per bounce [11, 12]. The simulation code also takes into account gravity affecting the vertical component of the UCN velocity. The most important parameters for a typical simulation run performed with the MCUCN code [29] adapted for this purpose are included in Fig. 1. The simulation starts with generation of UCNs with a uniform velocity distribution in a given velocity window emitted isotropically from the collimator entrance. At the collimator exit, UCNs form an almost parallel beam colliding with the moving detector surface along the beam axis.

An example simulation tracking $10^{7}$ neutrons with a uniform velocity distribution in the range $(0-8) \mathrm{m} / \mathrm{s}$ that enter the Ti collimator of the spectrometer was performed. Their histories were tracked until they were detected, absorbed in walls or returned back through the collimator. The oscillation amplitude and frequencies were set to $0.03 \mathrm{~m}$ and 5,10 and $15 \mathrm{~Hz}$, respectively. Table 1 collects corresponding velocity range for selected frequencies. The Fermi velocity of the detector material was chosen to be $4 \mathrm{~m} / \mathrm{s}$ (Fermi velocity of GS10 [27]).

The resulting velocity profiles for three different oscillation frequencies are shown in Fig. 6. As can be seen in the upper plot (corresponding to the highest achieved oscillation frequency of $15 \mathrm{~Hz}$ ) the neutrons with velocity lower than $1.2 \mathrm{~m} / \mathrm{s}$ are not transmitted and cannot be analyzed in this configuration. Similarly, the UCN with velocity higher than $7 \mathrm{~m} / \mathrm{s}$ have no window in the detector velocity distribution where they appear exclusively as can be seen by the characteristic small peak ("ear") on the high end of the distribution. This makes an unambiguous decomposition impossible. The missing UCN velocity intervals can be accessed by changing the oscillation frequency, the detector Fermi potential, e.g. via additional foils with different coatings, or both. 

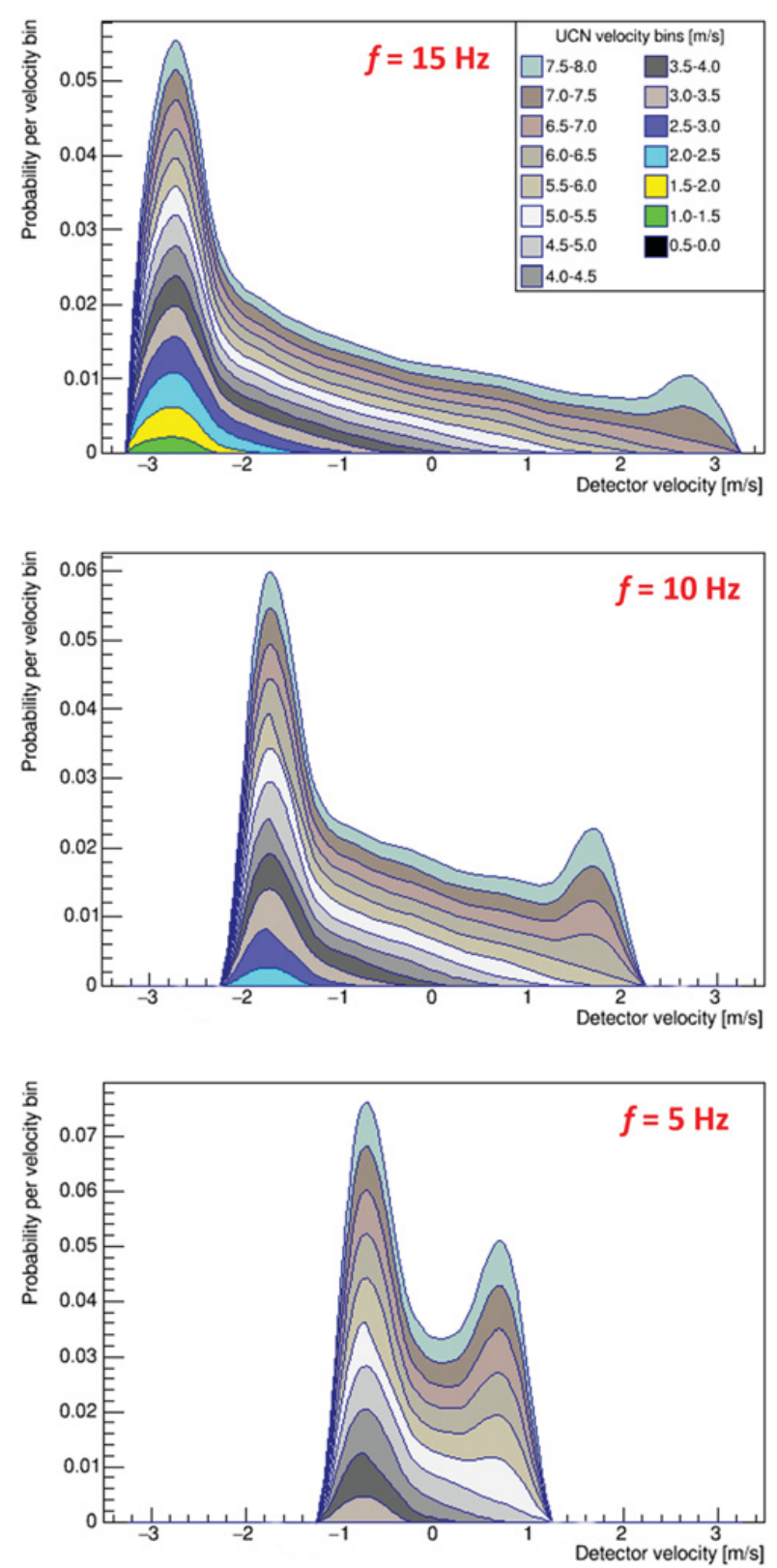

Figure 6. Detector velocity profiles corresponding to the UCN velocity bins for a uniform distribution of the initial velocity and with selected oscillation frequencies.

\section{Test measurement and preliminary analysis}

The first test measurements were performed at the West1 port of the PSI UCN source operating in a $300 \mathrm{~s}$ long cycle with $8 \mathrm{~s}$ beam pulses on the neutron spallation target. Therefore, the first few seconds of this data include a gamma flash and fast neutrons. For the rest of the cycle after the proton beam is turned off, the recorded UCN rate decreases as shown in Fig. 7.

Measurements were performed with three different oscillation frequencies: 5,10 and $15 \mathrm{~Hz}$ with $v_{\mathrm{d}}^{\mathrm{F}}=$ $4 \mathrm{~m} / \mathrm{s}$. In the first step of analysis, the detector counts corresponding to the $\gamma$ background were eliminated using the PSD relation defined in Eq. (8). Practically, only the signals with $Q_{\mathrm{L}}>0.8$ recorded by single SiPM sensors correspond to UCN detection as shown in Fig. 8. The gate

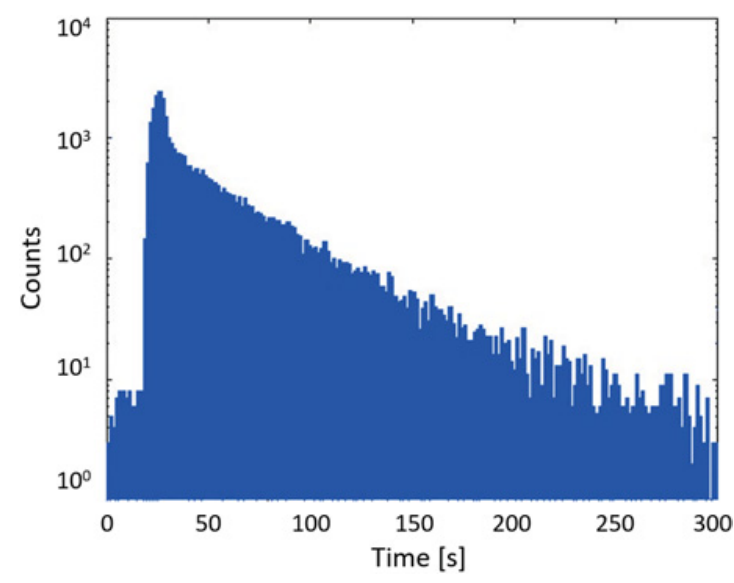

Figure 7. Neutron rate at the West-1 port as registered with the oscillating $\mathrm{UCN}$ detector $(f=15 \mathrm{~Hz})$. The proton pulse on the spallation target starts at time $=20 \mathrm{~s}$.

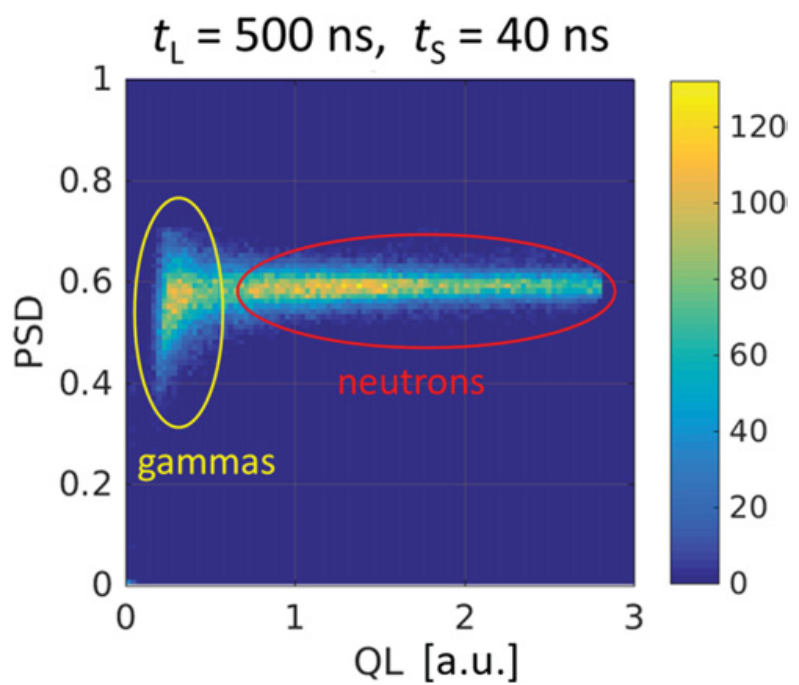

Figure 8. PSD as a function of $Q_{\mathrm{L}}$ from UCN data taken on the West- 1 beamport. The events are collected from the selected SiPM.The UCN events are located PSD in the region $0.8<Q_{\mathrm{L}}<$ 2.8 and $\gamma$-rays at $Q_{\mathrm{L}}<0.5$, respectively.

times were chosen according to the shape of the signals (see Fig. 4).

The selected neutron events are time-stamped and synchronized to the oscillation driving system so that the detector velocity at instant of detection can be assigned uniquely as described earlier. Figure 9 shows the resulting detected UCN distributions as a function of the detector velocity for three different oscillation frequencies. The qualitative similarity to the simulated distributions presented in Fig. 6 is clearly visible.

In the next step, the spectrum decomposition will be performed using the linear pseudo-inverse problem with Tikhonov regularization [30] according to the following equation:

$$
N_{i}^{\text {detected }}=\sum_{i j} F_{i j} N_{j}^{\mathrm{UCN}}
$$

where $N_{i}^{\text {detected }}$ is the number of UCNs detected in the $i$-th detector velocity bin, $F_{i j}$ is the value of the spectrometer response function obtained from $\mathrm{MC}$ simulation and $N_{j}^{\mathrm{UCN}}$ is the unknown number of UCN with velocity contained within $j$-th bin. The spectrometer response 

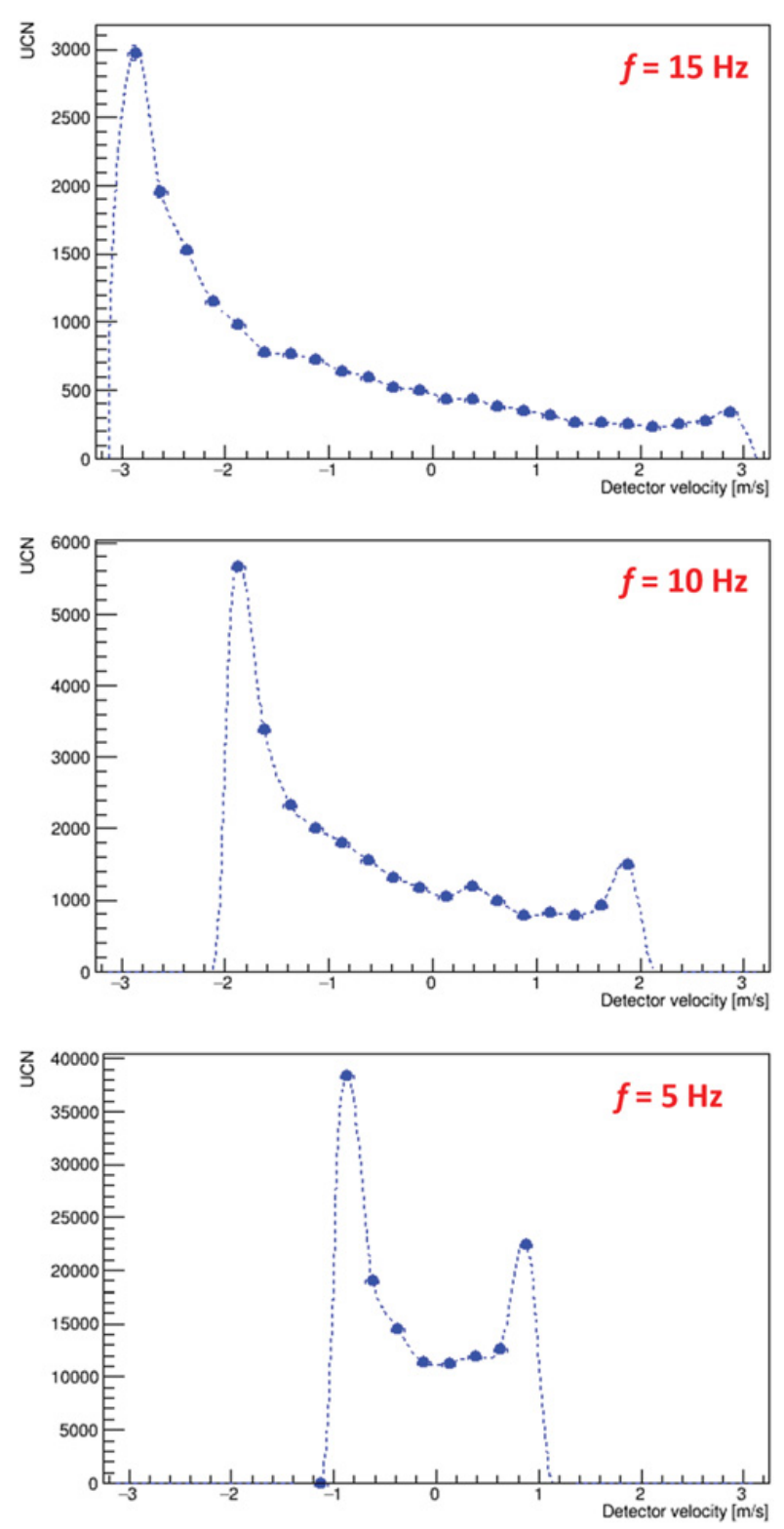

Figure 9. Detector velocity distributions extracted from a sample of data taken at West-1 port of the PSI UCN source.

function defines the probability that a neutron with the velocity $v_{n}$ entering the collimator will be registered by a detector moving with the velocity $v_{\mathrm{d}}$. For practical reasons it is devided into a set of detector velocity profiles corresponding to UCN velocity bins. It is important to note that "fast" neutrons (above $7 \mathrm{~m} / \mathrm{s}$ ) are clearly visible in the UCN velocity spectrum even for the highest oscillation frequency of $15 \mathrm{~Hz}$. This is indicated by an apparent "ear" at the high detector velocity side of the distribution. Further analysis of the spectrum evolution showed that this happens only in the first part of the UCN cycle (after the main pulse). Apparently the "fast" UCN die out quickly. Nevertheless, their presence in the experimental distributions prevents a unique decomposition (details of the decomposition will be covered in a future publication) and thus achieving the ultimate goal of the described project. It is necessary to cover the high UCN velocity range using either higher oscillation frequency or higher Fermi potential of the detector surface. This could not be achieved in the first test measurement.

\section{Conclusions and outlook}

The method proposed in this paper is based on the use of an oscillating detector with a time-stamping data acquisition system that allows for scanning the entire UCN velocity range in one oscillation cycle. Thanks to this it is possible to acquire a large part of the spectrum under the same conditions and study its evolution in time. The paper presents the first stage of the data analysis from the first test measurement made at the West-1 beamport of the PSI UCN source. After completion of the datataking (measuring also the "fast" UCN neutrons) the decomposition of the detector velocity distribution will allow to obtain the UCN energy spectrum. It is planned to carry out measurements with the different Fermi potential of the detector surface in order to study the UCN energy spectrum evolution.

We would like to thank the technical staff, in particular F. Burri and K. Lojek for their support during the construction of the spectrometer. We gratefully acknowledge the important work carried out by the mechanical workshop of the Institute of Physics of the Jagiellonian University. We also acknowledge the excellent support of the BSQ group operating the UCN source. This research was partly financed by the Polish National Center of Science grants: UMO-2016/23/D/ST2/00715 and UMO2015/18/M/ST2/00056.

\section{References}

[1] PSI UCN source; www.psi.ch/ucn/ultracoldneutron-source-ucn

[2] LANL UCN source; http://lansce.lanl.gov/ facilities/ultracold-neutrons

[3] FRM2 UCN source; ww. frm2.tum.de/en/theneutron-source

[4] TRIUMF UCN source; www.triumf .ca/ucn

[5] SNS fundamental neutron physics beamline; http://neutrons.ornl.gov/fnpb

[6] T. Soldner et al., EPJ Web of Conf. 219, 10003 (2019)

[7] PNPI-Gatchina UCN and cold neutron source; J. Phys. Conf. Ser. 798, 012147 (2017)

[8] O. Zimmer, R. Golub, Phys. Rev. C 92, 015501 (2015) [arXiv:1303.1944v2]

[9] NCSU UCN source; Nucl. Instrum. Meth. Phys. Res. A 767, 169 (2014)

[10] K. Mishima, JPS Conf. Proc. 22, 011033 (2018)

[11] R. Golub, Richardson, Lamoreaux, Ultra-cold Neutrons (Adam Hilger, Bristol, Philadelphia and New York, 1991)

[12] V.K. Ignatowitch, The Physics of Ultracold Neutrons, (Clarendon Press, Oxford, 1990)

[13] D. Dubbers and M.G. Schmidt, Rev. Mod. Phys. 83, 1111 (2011)

[14] H. Abele, Prog. In Part. And Nucl. Phys. 60, 1 (2008)

[15] J.S. Nico, W.M. Snow, Rev. Nucl. Part. Sci. 55, 27 (2005)

[16] A. Young et al., J. Phys. G: Nucl. And Part. Phys. 41, 114007 (2014)

[17] J. Jakubek et al., Nucl. Instrum. Meth. Phys. Res. A 600, 651 (2009)

[18] M. Lasakov et al., J. Res. Natl. Inst. Stand. Technol. 110, 287 (2005)

[19] P. Fierlinger et al., Nucl. Instrum. Meth. Phys. Res. A 557, 572 (2006) 
[20] A. Knecht, University of Zurich PhD Thesis (2009)

[21] D. Ries, ETH Zurich PhD Thesis (2016)

[22] S. Afach et al., Phys. Rev. Lett. 115, 162502 (2015)

[23] B. Lauss, Phys. Procedia 51, 98 (2014)

[24] S13360-6050PE; www.hamamatsu.com

[25] Applied Scintillation Technologies Ltd. company

[26] L. Göltl et al., Eur. Phys. J. A 49, 9 (2013)
[27] G. Ban et al., Eur. Phys. J. A 52, 326 (2016)

[28] DT5742; www.caen.it

[29] G. Zsigmond, Nucl. Instrum. Meth. Phys. Res. A 881, 16 (2018)

[30] H. Engl, M. Hanke, A. Neubauer, Regularization of Inverse Problems (Kluwer Academic Publishers, Dordrecht, Netherlands, 2000) 